\title{
Design of an electromagnetic mill. Its technological and control system structures for dry milling
}

\author{
Marta Wolosiewicz-Glab ${ }^{1, a}$, Dariusz Foszcz ${ }^{1}$, Tomasz Gawenda ${ }^{1}$, and Szymon Ogonowski ${ }^{2}$ \\ ${ }^{1}$ AGH University of Science and Technology, Faculty of Mining and Geoengineering, Department of Environmental \\ Engineering and Mineral Processing, Poland \\ ${ }^{2}$ Silesian University of Technology, Faculty of Automatic Control, Electronics and Computer Science, Institute of \\ Automatic Control, Poland
}

\begin{abstract}
A modern industry could not operate without complex processing of mineral raw materials, supported by innovative technologies. That is the reason to design and create a new electromagnetic mill. The paper presents a design of its technological structure and control system. It is presented that, in relation to traditional solutions, the electromagnetic mill is a fully automated system, which affects the effectiveness of its operation. The effectiveness and efficiency of processes depend on a proper selection of the physical parameters of the system and process parameters. The device is characterized by low energy consumption.
\end{abstract}

\section{Introduction}

Specified requirements for systems of grinding and classification, regarding the reduction of energy consumption, grinding, optimal particle size, particle shape and their surface properties showed that there is a need to design a modern fine grinding system with recirculation of grains which do not meet quality requirements. To meet the requirement of a competitive system, it should have a universal application, possible for all kinds of raw materials and configurable by appropriate measuring and control system and a dedicated application HMI/SCADA. An electromagnetic mill with the control system allows either dry or wet milling of raw materials in a closed cycle, with the possibility and very wide range, selection of speed of movement of the grinding media with different parameters. The ability to control the size of the dosing feed and grinding media, the ongoing analysis of particle size and status of the mill and the flow of recycle will enable much more effective mechanical activation of generated particles with specific physical and mechanical parameters (size, shape, surface area and energy, surface properties), and also significant increase in the grinding efficiency and energy savings. Heat losses in the working chamber will be used to simultaneously dry and heat the material to be processed and will increase the milling efficiency. The grinding process is usually only one stage of processing of materials, and the parameters of the ground product are closely related to the requirements of technological processes in the next stages. Not always the greatest possible degree of fineness of the material in the mill is required. The best results in the following process after grinding is being obtained for the certain particle size range. In addition, the parameters of the grinding process

${ }^{\text {a }}$ Corresponding author: wolosiewiczm@gmail.com 
are often subject to changes resulting from the technological situation of the whole production line. Sometimes it is necessary to change the parameters of mills due to the deteriorating performance of the flotation process of the milled material. Currently, the electromagnetic mill available on the market neither has a feeding-receiving system nor a dedicated control system.

\section{Electromagnetic mill - design}

The electromagnetic mill is a device in which the ferromagnetic grinding media are moved by deliberately generated rotating electromagnetic field, as an energy carrier. The basic elements of the mill are: inductor of rotating magnetic field and placed in its axis tube, forming a working chamber [1]. The electromagnetic mill was a little-known grinding machine used in laboratories rather than in industry. This limitation of applications results from the very low productivity and low efficiency of this type of mills. Low technical-motor parameters were influenced by improper design assumptions led to very large losses in the inductor windings of a rotating magnetic field. One of the advantages of the electromagnetic mill can be the possibility to enhance many processes by carrying out grinding in a region where, due to the electromagnetic field, ferromagnetic rods (grinding media) with a suitably chosen length to diameter ratio, rotate $[1,2]$.

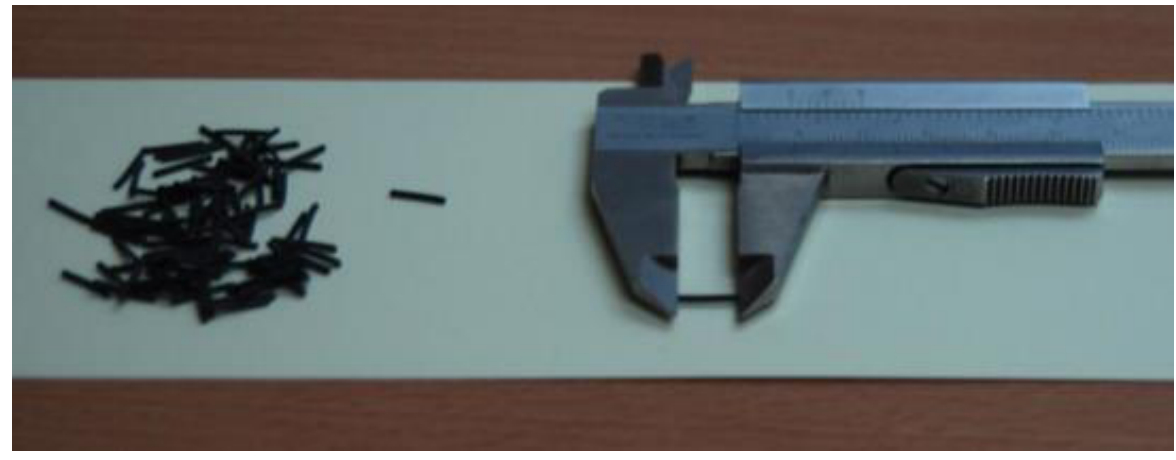

Figure 1. Grinding media used in electromagnetic mill (author's photo).

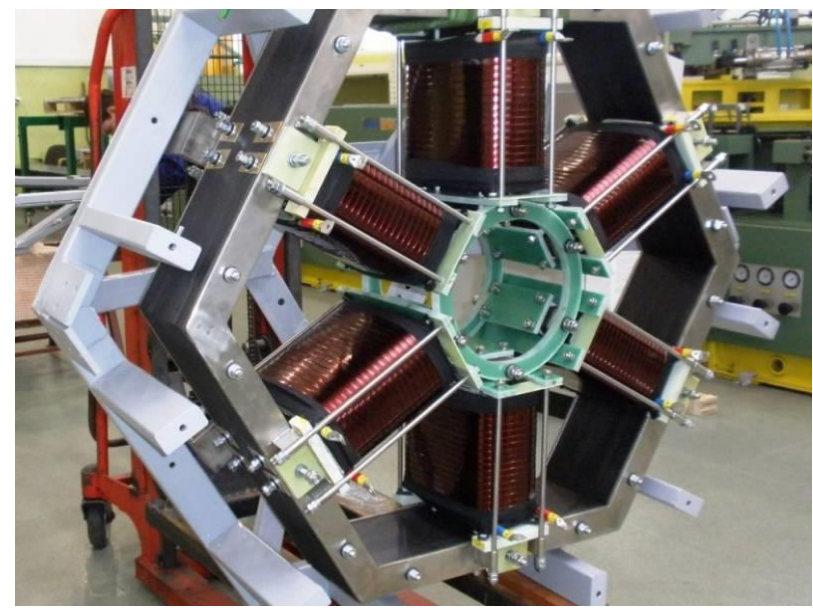

Figure 2. Magnetic inductor developed by ELTRAF company. 
In contrast to conventional mills (ball, rod, or cylpebs) the mill housing is stationary while the grinding (or mixing) takes place in the working chamber, inside which move small ferromagnetic elements - called grinding media. Their movement is caused by the action of the vortex electromagnetic field $[2,3]$. The effectiveness and efficiency of the process carried out in the mill are dependent on the proper selection of the physical parameters of the structure and process parameters such as the flow rate of feed (residence time), particle size of the input, speed and intensity of the rotating electromagnetic field, temperature, humidity, and other parameters of the workpiece (e.g. lithological composition) [1].

A basic condition for industrial use of electromagnetic mill is its continuous operation. Grinding media should be selected for the particle size of the particulate material. In the case of large variation in the material grain size, a blend of grinding media of different sizes can be applied. Any change in the size of the grinding media can be adapted to the physical properties of the ground material and the desired final fineness of the ground product [2, 3]. Defined requirements for grinding and classification systems related to the reduction of energy consumption of grinding, optimum grain size, grain shape and their surface properties showed that there was a need to design a modern fine grinding system with recirculation of grains which do not meet quality requirements. To make this solution competitive, system should be universal for possible all kinds of raw materials, configurable using a measuring and control system as well as a dedicated HMI/SCADA software. Such requirements impose a need for mills with a unique operating principle and the wide possibilities of parameterization of its work. As part of the SCADA system, the following optimization problems will be solved:

- maximization of the processing throughput with the limitation on the quality of the product,

- minimizing the energy consumed in grinding at a specified throughput, and

- maximization of the degree of fragmentation.

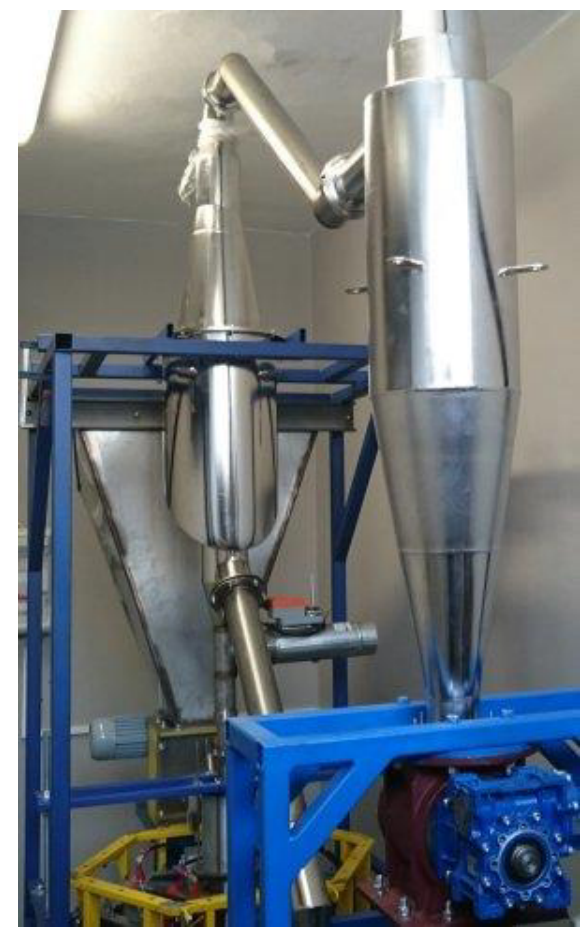

Figure 3. Classifiers close-up. 
Decision variables of these tasks will be the defining points of the mill work (gear motor output, product temperature, etc.). Arguments of the criterion functions arise from the evaluation of the quality of the process, such as the parameters of the particle size distribution, average or borderline grain size, the total value of the electricity for the processing, etc. To determine the values of decision variables from these arguments, the suitable polynomial models and artificial neural networks will be identified [4]. Such requirements impose a need for mill with a unique operating principle and the wide possibilities of parameterization of its work. The SCADA system will record parameters i.e. the quality parameters defined as the design criterion function optimization tasks and the process parameters, either measured or adjusted during grinding for a single charge. On this basis, the SCADA system on a regular basis will perform principal component analysis (PCA). On the basis of the principal component scatter, the criterion indication of the deregulation process of batch grinding will be developed. There will be developed automatic decision-making procedures in the event of deregulation.

\section{Design of the technological system}

The processes of mechanical comminution are characterized by enormous energy intensity, that is why in this direction intensive research on modern design solutions of machines and systems for shredding and classification are carried out. Shredding processes are widely used in various fields of material processing to obtain finished products, e.g. mineral aggregates with a suitable grain size, sorbent limestone, cement. The most important characteristics of the milling process include:

- producing much larger quantities of the smallest particle size fraction,

- a much higher energy consumption per unit of mass-processed raw material (technological efficiency)

- higher particles fineness than in the processes of crushing,

- a large part of abrasive action in most of these processes,

- high wear of working elements per unit of processing,

- higher than in other processes probability of producing a certain amount of undersize particles.

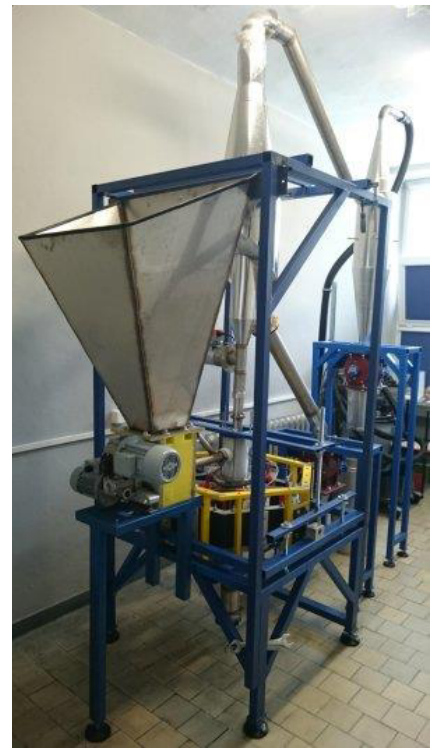

Figure 4. Electromagnetic mill and classifiers. 
The final product coming out from an accurate classifier is separated from the transporting air in the cyclone and goes into the final product tank. In addition, the installation is equipped with a cooling system of the working chamber in the form of a set of fans with controlled output. The concept of the installation is shown in Fig. 5 which depicts the chosen measuring points parameters such as speed, pressure, humidity and air temperature, temperature and humidity of the material, mass of material in individual tanks, position of the flaps and engine speeds.

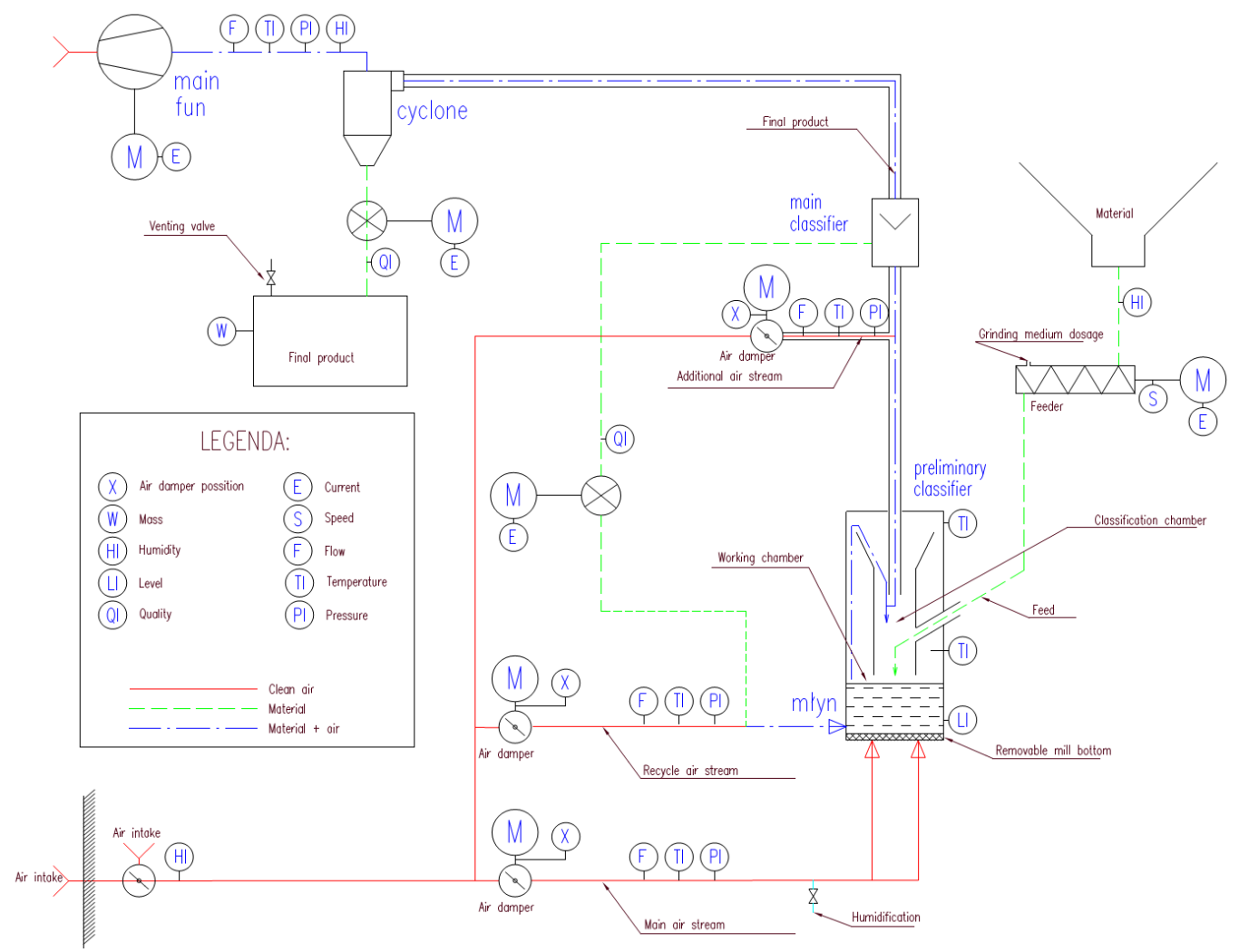

Figure 5. Functional structure of dispensing system of grinding media along with classification system [5].

\section{Design of the control system}

The installation of the electromagnetic mill is a complex system with many control goals defined for each subsystem. Obtaining the output with required particle size, which is the main objective of the control system, is carried out during the operation of the mill under nominal conditions, which means after startup procedures, constituting a separate group of tasks performed by the control system. Additional supervisory objectives of the control system in the electromagnetic mill is to obtain the required temperature and humidity of the product. The vertical arrangement of the mill causes the control system of air streams fed to the mill system is designed to keep the ground material in the working chamber. For a better tightness of the transport system it was decided to use a vacuum system with a single pulling fan as a major source of air flow as shown in Figure 6.

Therefore, the control system uses flaps regulating the flow of a stream of primary air in the pipes, recycle and secondary air, as actuators of control systems, keeping ground material in the working chamber. This results in a significant complication of the control system, because any change in the 
position of any flap, associated with the reaction of the local control system changes the flow of the remaining air streams $[4,6]$.

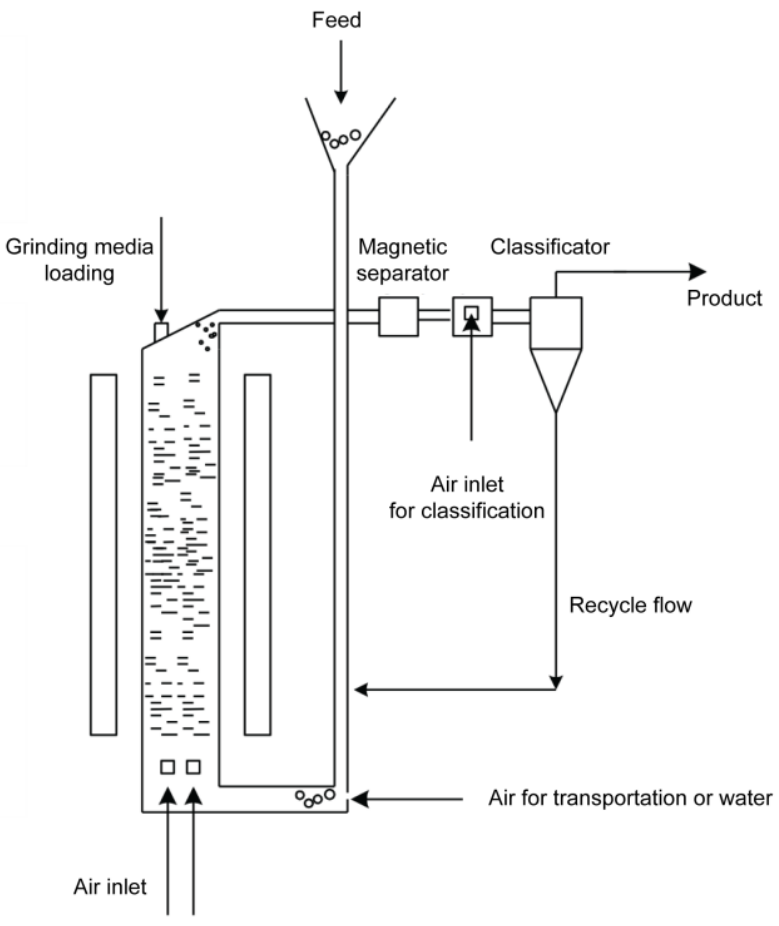

Figure 6. Dry and wet milling in vertical layout [5].

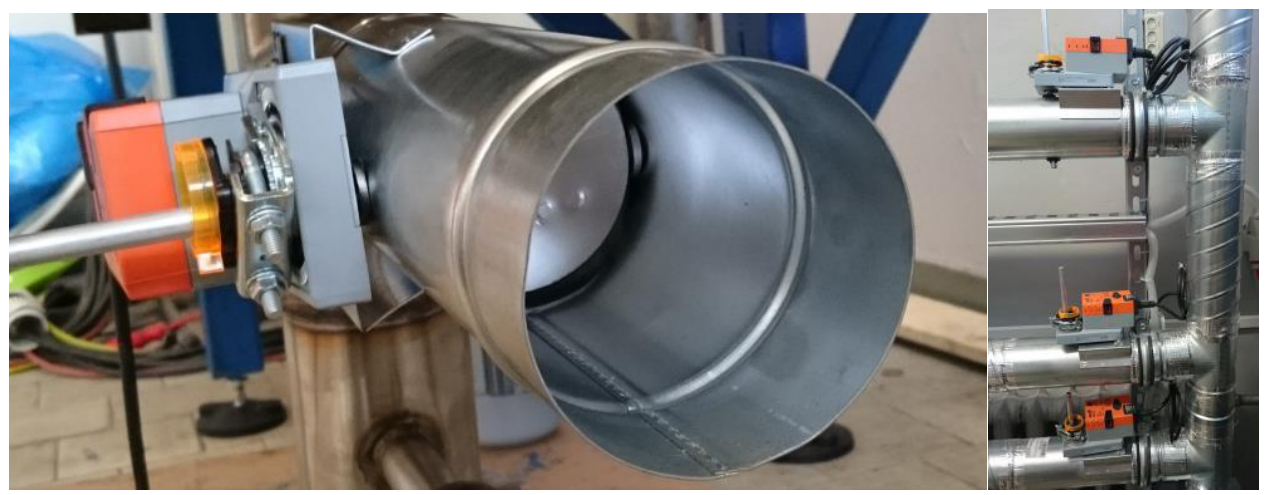

Figure 7. Flaps regulating flow of stream of primary air, recycled air and secondary air .

Another task of the control system is to stabilize the mixture of grinding media and ground material as well as to control the rotating electromagnetic field. The initial studies showed that changing mentioned process parameters significantly affected the fineness of the product. All parameters mentioned above are interrelated, meaning that the control object is multi-dimensional and has a strong internal cross-coupling feedback. From the point of view of the design of the control system, a plurality of the above-mentioned quantities (measured, adjusted and state) were divided into 
control, disturbance and output signals (Fig. 8). This approach not only allows the systematization of each quantities in the system, but also modelling the object for the control purposes [7].

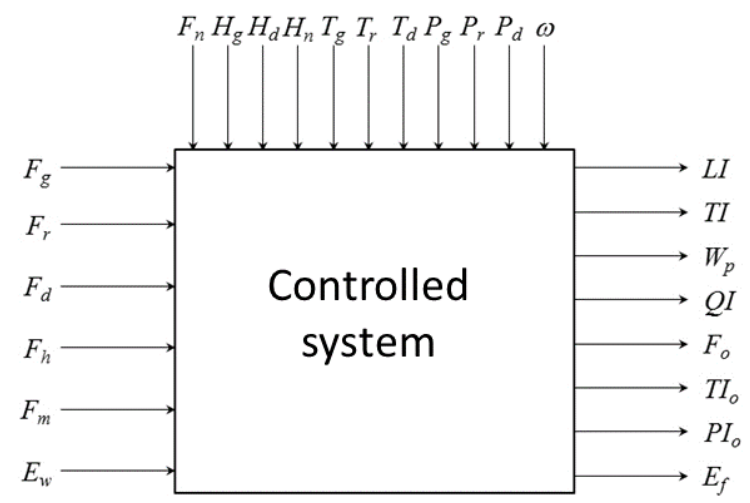

Figure 8. General structure of the electromagnetic mill installation as a controlled system.

Control quantities are: primary air speed $F_{g}$, recycle air speed $F_{r}$, additional air speed $F_{d}$, volumetric flow of water mist to feed $F_{h}$, weight stream of grinding media $F_{m}$ and voltage control of cooling fans $E_{w}$. Disturbance variables are: weight feed stream $F_{n}$, humidity of air in primary stream $H_{g}$, air humidity in the additional stream $H_{d}$, initial feed humidity $H_{n}$, temperature of air in the primary stream $T_{g}$, temperature of air in the recycle stream $T_{r}$, air temperature in an additional stream $T_{d}$, air pressure in primary stream $P_{g}$, air pressure in recycle stream $P_{r}$, air pressure in an additional stream $P_{d}$, frequency of rotating electromagnetic field $\omega$ as well as grain composition of feed and susceptibility to crushing of the material during the shredding process. The output quantities are: working chamber filling level $L l$, average temperature of preliminary classifier $T I$, weight of product in product tank $W_{p}$, product quality $Q I$, speed of exhaust air $F_{o}$, temperature of exhaust air $T I_{o}$, pressure of exhaust air $P I_{o}$, load current of inverter generating rotating field $E_{f}$.

The classification was the starting point for the design of the functional structure of control systems implementing the objectives described above. These objectives are carried out through the implementation of specific tasks in the control system. These tasks, however, are carried out within the framework of unequal time horizons. Moreover, they are interconnected by the multidimensionality of the plant, and through cross-compliance control purposes. It was essential to implement the control system with hierarchical structure [4], which general overview is presented in Fig. 9.

For each of the subsystems, specific tasks were formulated, carried out by their local control systems. These tasks were defined by the states of the subsystems to be controlled. Basic tasks of direct control are [7, 10]:

- stabilization of speed of the air flow in the working chamber in open and in recycle circuit,

- stabilization of speed of the air stream at the input of accurate classifier,

- control of grinding media dispenser,

- adjust the degree of filling of the working chamber,

- feed material humidity control,

- stabilization of product moisture,

- stabilization of the temperature in the working chamber. 


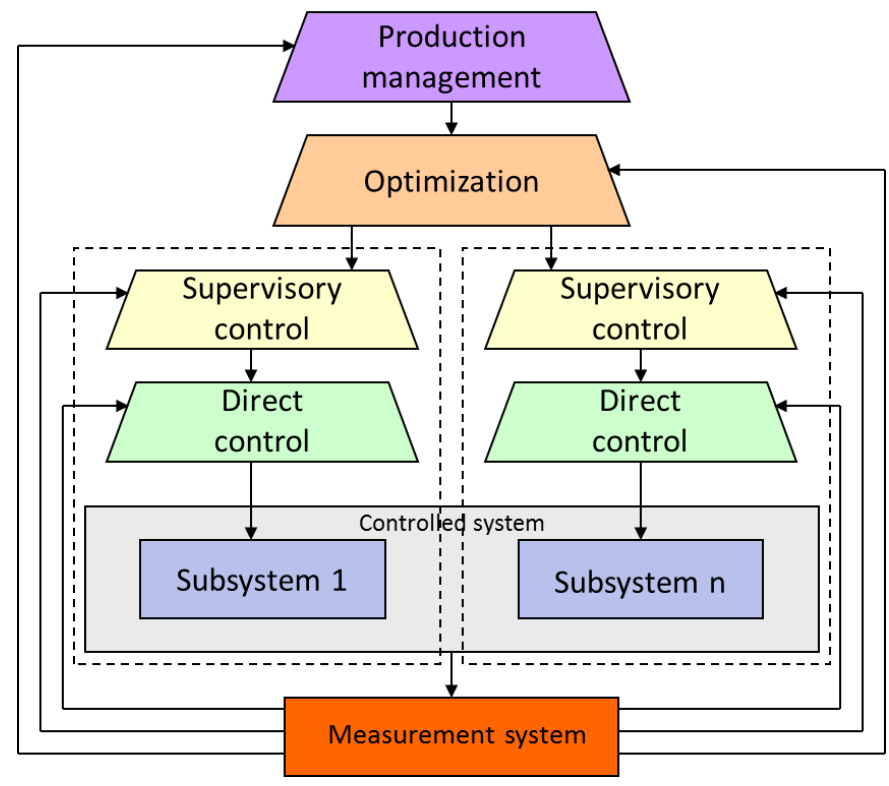

Figure 9. General structure of the multi-layered control system.

The tasks of the supervisory control layer concern, inter alia, the determination of the set point correction for air flow regulators depending on such parameters as humidity and temperature of the feed and air; set point correction control of the primary stream regulator, depending on the recycle state; determining a set point for additional flow controller; startup sequences management; the preand post-startup control of actuator elements in the installation [11].

The task of optimization control layer is to determine the operating points of the electromagnetic mill. The most important operating point of the mill is its load - the mass flow of the feed. For the supervisory control layer, as well as for the direct layer, the feed stream is the leading quantity, which is the basis for design of the functional structure of control systems in these layers. In general, the task for optimization layer is to determine the operating parameters of the mill, which minimize energy consumption with the restrictions arising from the required product quality, expressed by the granulation. For the optimization task, also other constraints such as temperature and humidity of the product and the geometric properties of grain, can be taken under consideration:

The primary objective of the measurement system in the electromagnetic mill is to measure all physical quantities necessary to implement the mill control algorithm. The measurement system should enable the measurement of physical quantities associated with all media streams present in the system.

Implementation of the control system assumes the use of SIMATIC S7-300 controller with digital and analog I/O modules for direct and basic supervisory control. All analog measurement and control signals were brought to the $4.20 \mathrm{~mA}$ standard, which provided a lower susceptibility to disturbances. Direct control with the use of inverters will be executed in an additional PLC system with SIMATIC S7-1200 controller, which also provides the MODBUS communication standard [12]. The communication between the controllers will take place in the PROFINET network. The tasks of the supervisory layer are implemented in PLC controllers and in SCADA IFIX system installed on the operator panel SIMATIC IPC. For the purpose of the data processing for soft-sensing system [12] using basic measurement values and additional quality measurements (eg. the vision system 6), PC computer was installed, connected with the panel via Ethernet network. Additionally, for the registration of data from acoustic [11, 10] and vibration sensors [9] Dataloger (DLAS), was directly connected to the operator panel. 


\section{Conclusions}

The article presents the construction of an innovative electromagnetic mill, which compared to traditional solutions provide a significant reduction in energy consumption and higher technological performance. The device is equipped with a control system, either measuring the grinding quality or analyzing the operating status of the mill. Direct control of the grinding process is implemented in industrial controllers (PLC) and is supported by the optimization algorithms that run in the supervisory control system (SCADA). Designed during the project low-cost way of the real time measuring of particle size of the feed and products, as well as flow classification, can be used in any field of mineral processing, not only related to the grinding in the electromagnetic mill, in which there are similar processes (crushing and grinding, screening, hydraulic and air classification, flotation and gravity enrichment), but also in cement and lime industry and in energy sector.

\section{Acknowledgement}

Development of the article was funded in the framework of the project: PBS3/B3/28/2015

\section{References}

1. M. Wolosiewicz-Glab, D. Foszcz, in Proceedings of $5^{\text {th }}$ European Young Engineers Conference (Warsaw University of Technology, 117, 2016)

2. M. Wolosiewicz-Glab, D. Foszcz, in Proceedings of $5^{\text {th }}$ European Young Engineers Conference (Warsaw University of Technology, 117, 2016)

3. M. Wolosiewicz-Glab, D. Logistyka, 4, 9930 (2015)

4. Z. Ogonowski, Appl. Energy, 88, 1586 (2011)

5. M. Pawelczyk, Z. Ogonowski, Sz. Ogonowski, D. Foszcz, D. Saramak, T. Gawenda, Patent No. P.413041 (6.07.2015)

6. Z. Ogonowski, in Proceedings of $19^{\text {th }}$ International Conference on Process Control (High Tatras, Slovak Rep., 2013)

7. Sz. Ogonowski, Energy Build., 42, 1510 (2010)

8. P. Tatjewski, Advanced Control of Industrial Processes: Structures and Algorithms (SpringerVerlag, London, 2007)

9. P. Kadlec, B. Gabrys, S. Strandt, Comp. Chem. Eng., 33(4), 795 (2009)

10. A. Konieczny, M. Kurzydlo, Sz. Ogonowski, D. Foszcz, in Proceedings of the Mineral Engineering Conference MEC 2014 (Istebna, 2014)

11. S. J. Spencer, J. J. Campbell, K. R. Weller, Y. Liu., IPMM, ‘99(2), 939 (1999)

12. M. Kurzydlo, M. Pawelczyk, Vibroeng. Procedia, 6, 18, (2015) 